\title{
Ecologia do forrageio por Cyphomyrmex morschi Emery (Hymenoptera, Formicidae) em vegetação de restinga no Sul do Brasil
}

\author{
Benedito Cortês Lopes
}

Departamento de Ecologia e Zoologia, Universidade Federal de Santa Catarina. Caixa Postal 476, 88010-970 Florianópolis, Santa Catarina, Brasil. E-mail: bclopes@ccb.ufsc.br

\begin{abstract}
Foraging ecology of Cyphomyrmex morschi Emery (Hymenoptera, Formicidae) in sand dune vegetation at Southern Brazil. A total of 400 nests of Cyphomyrmex morschi Emery, 1887 was evaluated between 1997 and 1998 at the dunes of the Joaquina Beach, Florianópolis, Santa Catarina, in order to verify the substrate brought back to the nests. These ants use vegetable or animal material (excrements of lepidopteran larvae or carcasses of beetles or ants) or even not identified material that are used to culture the fungus. Thus, ecologically speaking, $C$. morschi can be considered a detritiphagous species.
\end{abstract}

KEY WORDS. Fungus-growing ant; resources; Santa Catarina Island; Attini.

RESUMO. Foram amostrados 400 ninhos de Cyphomyrmex morschi Emery, 1887 entre 1997 e 1998, nas dunas da praia da Joaquina, Florianópolis, SC, para a verificação do material transportado ao ninho. Estas formigas utilizam material de origem vegetal ou animal (fezes de lagartas de Lepidoptera ou partes de corpos de besouros ou formigas) ou mesmo material não identificado que são introduzidos no ninho para o cultivo do fungo. Assim, do ponto de vista do papel ecológico desempenhado, pode-se considerar $C$. morschi como uma espécie detritófaga.

PALAVRAS-CHAVE. Formigas cultivadoras de fungo; Ilha de Santa Catarina; recursos utilizados; Attini.

Devido ao impacto econômico causado a diversas monoculturas, aspectos da ecologia de formigas cortadeiras dos gêneros Acromyrmex Mayr, 1865 e Atta Fabricius, 1804 já foram estudados e até quantificados (Rockwood 1976, Fowler et al. 1986, WIRTH et al. 1997).

Por outro lado, os demais gêneros que compõem a tribo Attini têm sido menos estudados, apesar da importância que possam ter para a discussão da evolução do cultivo de fungos nesse grupo deformigas (Weber 1972, HöLldobler \& Wilson 1990, Mueller \& W Cislo 1998, Brandão \& Mayhé-Nunes 2001).

Assim, estudos envolvendo a ecologia de forrageio das formigas cultivadoras de fungos são relevantes, no sentido que revelam os substratos levados aos formigueiros e que servirão de meio para o desenvolvimento do fungo simbiôntico, fonte de alimento das colônias (Howard 1991, MuraKamı \& HIGASH 1997). Outra especulação que pode ser tirada da ecologia de forrageio em Attini diz respeito à posição filogenética dos 12 gêneros (Brandão \& MaYHÉ-Nunes 2001), sendo que aqui há dois pontos de vista contrastantes: um queaceita o gênero Cyphomyrmex Mayr, 1862 como o mais basal (ou "primitivo") dentro da tribo (WEBER 1972, HöLLDOBLER \& WILSON 1990) eoutro que diz queCyphomyrmex tem uma posição intermediária na tribo (KusNEzov 1963, SchuLtz \& Meier 1995, Mayhé-Nunes \& Jaffé 1998, Currie et al. 1999).

Os objetivos do presente trabal ho foram: verificar os subs- tratos transportados aos ninhos por operárias de Cyphomyrmex morschi Emery, 1887, verificar se há variações sazonais no uso destes recursos e acrescentar informações para a discussão filogenética do gênero Cyphomyrmex dentro da tribo Attini.

\section{MATERIAL E MÉTODOS}

O estudo foi realizado na restinga da Praia da Joaquina (27은 40"S, 48ำ27'10"W), Florianópolis, Santa Catarina. Esta praia do setor sudoeste da IIha de Santa Catarina tem cerca de $3 \mathrm{~km}$ de comprimento e apresenta, do mar para o interior, uma série de dunas fixas cobertas por vegetação herbácea e arbustiva, seguida de baixadas onde se encontram lagos permanentes e depressões úmidas, circundadas por dunas de pequeno porte (CAstellanı et al. 1996). O clima geral é do tipo Cfa, segundo a classificação de Koeppen, isto é, mesotérmico úmido, sem estação seca definida e com verão quente (SAnTos et al. 1997).

Cyphomyrmex morschi é uma formiga relativamente pequena (3,0 mm), com operárias monomórficas e descrita originalmente de material proveniente de São Lourenço, Rio Grande do Sul (KEMPF 1964). É uma espécie que ocorre em áreas litorâneas, desde a Argentina até a cidade de Cabo Frio, Rio de Janeiro (KempF 1964, Bonnet \& Lopes 1993). Apesar de não haver registro para o litoral do Paraná, muito provavelmente esta espécie também ocorra nesse estado.

Revista Brasileira de Zoologia 24 (1): 52-56, março 2007 
O ninho dessa espécie é caracterizado por uma pequena abertura no solo rodeada de uma cratera de terra ou areia solta (Von IHeRING 1894).

Entre 1997 e 1998 foram analisados 400 ninhos de Cyphomyrmex morschi, encontrados al eatoriamente, 50 ninhos por estação, com 10 minutos de observação por ninho, sempre no período diurno. Para cada um, era feita a contagem do número de operárias que saíam para forragear, quantas dessas voltavam e, quando possível, era feita a identificação visual do material trazido. Devido ao hábito tímido e ao comportamento de se fingirem de mortas quando tocadas (tanatose), adotou-se apenas a observação visual, para evitar alterações no comportamento das operárias.

Para a identificação do material transportado aos ninhos, foi montado um herbário para comparações com o material vegetal depositado no Herbário FLOR, Departamento de Botânica, Universidade Federal de Santa Catarina.

Para a comparação dos recursos, a nível sazonal, usaram-se os índices de diversidade de Simpson (cada listagem de material usado por estação é considerada uma agregação ou comunidade diferente) e o de similaridade de Morisita. Para avaliar a significância dos valores obtidos com o índice de Simpson foi aplicado o teste t (Brower et al. 1998).

\section{RESULTADOS}

Dos 400 ninhos observados, foi verificado o forrageio em 145 dos 200 do ano de 1997 e em 156 dos 200 de 1998. Nos ninhos sem forrageio, as operárias estavam inativas durante a observação ou realizando atividades de desobstrução das galerias.

Devido ao pequeno tamanho das operárias e ao método de observação, foi grande a taxa de "material não identificado" transportado aos ninhos, bem como "material vegetal não identificado" (Tab. I). A diferença entre essas categorias é que, no segundo caso, foi possível apenas verificar a origem vegetal do substrato carregado. Para os demais casos, "material vegetal identificado" (discriminado na Tab. I) e "material de origem animal" (fezes de lagartas de lepidópteros ou pedaços de corpos de insetos), os valores foram menores.

Nos dois anos de observação, foi grande a proporção de recursos de origem vegetal: $59,9 \%$ em 1997 , sendo $41 \%$ não identificado e $18,9 \%$ identificado e $59,7 \%$ em 1998, sendo $37 \%$ não identificado e 22,7\% identificado. Asoperárias de C. morschi procuram por pedaços de folhas, folíolos, flores secas, pedaços de cascas e sementes caídas e misturadas na areia próxima ao ninho. Alguns desses materiais também são coletados em área de lixo de Acromyrmex striatus (Roger, 1863).

Do material vegetal identificado, os principais foram folíolos secos e flores secas de Stylosanthes viscosa Swartz (Leguminosae) (Tab. I).

O material de origem animal, representado principalmente por fezes de lagartas de Lepidoptera, partes de corpos de formigas ou de besouros, teve uma representação menos expressiva, 5,1\% em 1997 e 4,3\% em 1998.
Por outro lado, também foram expressivos os valores de material não identificado, 35,2\% em 1997 e 36,0\% em 1998. Se, em uma hipótese extrema, todo esse material não identificado fosse de origem animal, mesmo assim, os valores hipotéticos chegariam a 40,3\% em 1997 e 1998, o que continua apontando para porcentagens maiores de uso de material vegetal, identificado ou não.

Pelos valores do índice de diversidade de Simpson, se aceita a Ho de que as amostragens de recursos utilizados por C. morschi, estação por estação em 1997, provêm de agregações vegetais com a mesma diversidade (Tab. II). Por outro lado, em 1998, só não se aceita a Ho na comparação entre os valores de outono e inverno (teste t a $0,05[\infty]=1,96<$ t obtido $=2,29$ ).

Os valores do índice de similaridade de Morisita, entre cada estação dos anos de 1997 e 1998 e entre os anos estão na tabela III. Em todos os casos, as similaridades foram altas, com valores entre 0,96 e 1,01 .

\section{DISCUSSÃO}

O entendimento do uso de recursos por $\mathrm{C}$. morschi passa por uma análise comparativa dos substratos usados para a fungicultura por outras espécies de Attini consideradas “inferiores" ou não cortadeiras, indo de Cyphomyrmex até Trachymyrmex Forel, 1893. Assim, o substrato trazido para o ninho deveria apresentar uma gradação, desde fezes e corpos de insetos em Cyphomyrmex até matéria vegetal morta e partes de flores em Trachymyrmex (WEBer 1972, Hölldobler \& WiLSON 1990). O que provavelmente aconteceu, ao longo de décadas, foram observações esporádicas e pontuais que não quantificaram os substratos utilizados por esses gêneros menos conspícuos de Attini, quando em comparação com Acromyrmex e, principalmente, Atta (LEAL \& Oliveira 2000).

Recentemente tem havido uma série de trabal hos sobre a ecologia do forrageio em Attini não cortadeiras. Nas espécies estudadas, destaca-se a idéia de que é maior o uso de substratos vegetais em diversos graus de decomposição e menor o uso de substratos de origem animal (TORRES 1989, WALLER 1989, Murakami \& Higashi 1997, Leal \& Oliveira 1998, 2000, Mueller \& W CIslo 1998). Essa diferença de uso de substratos também foi verificada no presente estudo, uma vez que C. morschi apresentou cerca de $60 \%$ do seu material forrageado de origem ve getal e apenas de 4 a $5 \%$ de origem animal.

Assim como C. morschi coletou principalmente folíolos secos e flores secas de Stylosanthes viscosa (Tab. I), LeAL \& OLIVEIRA (2000) encontraram que mais de $95 \%$ dos registros de partes vegetativas eram foliólulos de Anadenanthera falcata (Benth.) Speg., também Leguminosae. Assim, esta família de planta parece apresentar algumas espécies com grande preferência por parte de Attini. No caso de Attini “inferiores" talvez essa preferência por Leguminosae possa ser devida, além dos aspectos químicos e nutricionais, pelas características físicas e tamanho dos folíolos e foliólulos caídos, fáceis de serem carregados por operárias que não cortam folhas de maior porte.

Revista Brasileira de Zoologia 24 (1): 52-56, março 2007 
Tabela I. Recursos utilizados por Cyphomyrmex morschi na Praia da Joaquina, Florianópolis, Santa Catarina, em 1997 e 1998. Número de vezes em que a planta ou o material foi utilizado, por estação do ano. (V) verão, $(\mathrm{O})$ outono, $(\mathrm{I})$ inverno, $(\mathrm{P})$ primavera e $(\mathrm{T})$ total anual.

\begin{tabular}{|c|c|c|c|c|c|c|c|c|c|c|}
\hline \multirow{2}{*}{ Recursos } & \multicolumn{5}{|c|}{1997} & \multicolumn{5}{|c|}{1998} \\
\hline & $\mathrm{V}$ & $\mathrm{O}$ & $\mathrm{I}$ & $\mathrm{P}$ & $\mathrm{T}$ & $\mathrm{V}$ & $\mathrm{O}$ & $\mathrm{I}$ & $\mathrm{P}$ & $\mathrm{T}$ \\
\hline Material não identificado & 14 & 21 & 23 & 24 & 82 & 26 & 34 & 22 & 18 & 100 \\
\hline Material de origem animal & 2 & 2 & 5 & 3 & 12 & 3 & 3 & 3 & 3 & 12 \\
\hline Material vegetal não identificado & 21 & 28 & 18 & 28 & 95 & 30 & 30 & 15 & 28 & 103 \\
\hline Folha de Stylosanthes viscosa (Leguminosae) & 3 & 3 & 6 & 7 & 19 & 8 & 6 & 8 & 10 & 32 \\
\hline Semente de gramínea & 2 & - & - & - & 2 & - & - & - & - & - \\
\hline Material em lixo de Acromyrmex striatus & 1 & 3 & 1 & - & 5 & - & 1 & 1 & - & 2 \\
\hline Folha de gramínea & 2 & - & 1 & - & 3 & - & - & - & - & - \\
\hline Flor de Chloris sp. (Gramineae) & 1 & - & - & - & 1 & - & - & - & - & - \\
\hline Semente de Chloris sp. (Gramineae) & 4 & - & - & - & 4 & - & - & - & - & - \\
\hline Líquen & 1 & - & - & 2 & 3 & 1 & - & 1 & - & 1 \\
\hline Semente de N oticastrum malmei (Compositae) & - & 1 & - & - & 1 & - & - & & - & - \\
\hline Semente de Paspalum sp. (Gramineae) & - & 1 & - & - & 1 & - & - & & - & - \\
\hline Semente não identificada & - & - & - & 2 & 2 & - & - & 1 & - & 1 \\
\hline Flor seca não identificada & - & - & - & 1 & 1 & - & - & & - & \\
\hline Folha seca de Polygala cyparissias (Polygalaceae) & - & - & - & 2 & 2 & 1 & - & - & - & 1 \\
\hline Folha de Cardionema ramosissima (Caryophyllaceae) & - & - & - & - & - & 1 & - & - & - & 1 \\
\hline Anteras de Paspalum sp. (Gramineae) & - & - & - & - & - & 1 & - & - & - & 1 \\
\hline Casca de semente não identificada & - & - & - & - & - & 1 & - & - & - & 1 \\
\hline Semente de Compositae & - & - & - & - & - & 1 & 1 & - & - & 2 \\
\hline Semente de Panicum sp. (Gramineae) & - & - & - & - & - & 1 & - & - & - & 1 \\
\hline Flor seca de N oticastrum malmei (Compositae) & - & - & - & - & - & - & 2 & - & - & 2 \\
\hline Flor seca de Acicarpha spathulata (Calyceraceae) & - & - & - & - & - & - & 1 & - & - & 1 \\
\hline Folha seca de N oticastrum malmei (Compositae) & - & - & - & - & - & - & - & 2 & 2 & 4 \\
\hline Flor seca de Euphorbia sp. (Euphorbiaceae) & - & - & - & - & - & - & - & 1 & - & 1 \\
\hline Flor seca de Dodonaea viscosa (Sapindaceae) & - & - & - & - & - & - & - & 1 & - & 1 \\
\hline Folha seca não identificada & - & - & - & - & - & - & - & 2 & 2 & 4 \\
\hline Folha seca de Paepalanthus polyanthus (Eriocaulaceae) & - & - & - & - & - & - & - & 1 & - & 1 \\
\hline Flor seca de Polygala cyparissias (Polygalaceae) & - & - & - & - & - & - & - & - & 2 & 2 \\
\hline Semente de Polygala cyparissias (Polygalaceae) & - & - & - & - & - & - & - & - & 2 & 2 \\
\hline Flor de Panicum sp. (Gramineae) & - & - & - & - & - & - & - & - & 1 & 1 \\
\hline Total de registros & 51 & 59 & 54 & 69 & 233 & 74 & 78 & 58 & 68 & 278 \\
\hline Número de espécies/materiais & 10 & 7 & 6 & 8 & 15 & 11 & 8 & 12 & 9 & 23 \\
\hline
\end{tabular}

Tabela II. Valores de diversidade de Simpson para os recursos usados por Cyphomyrmex morschi nos anos de 1997 e 1998 , Praia da Joaquina, Florianópolis, Santa Catarina.

\begin{tabular}{|c|c|c|c|c|c|c|c|c|}
\hline & \multicolumn{4}{|c|}{1997} & \multicolumn{4}{|c|}{1998} \\
\hline & Verão & Outono & Inverno & Primavera & Verão & Outono & Inverno & Primavera \\
\hline Índice de Simpson & 0,74 & 0,64 & 0,68 & 0,70 & 0,70 & 0,65 & 0,76 & 0,73 \\
\hline \multicolumn{9}{|l|}{ Teste t } \\
\hline Verão & - & 1,66 & 0,95 & 0,73 & - & 0,96 & 1,34 & 0,76 \\
\hline Outono & - & - & 0,83 & 1,12 & - & - & $2,29 *$ & 1,74 \\
\hline Inverno & - & - & - & 0,27 & - & - & - & 0,62 \\
\hline
\end{tabular}

(*) Teste t significativo a 0,05\%.

Revista Brasileira de Zoologia 24 (1): 52-56, março 2007 
Tabela III. Valores de similaridade de Morisita para os recursos usados por Cyphomyrmex morschi nos anos de 1997 e 1998 , Praia da Joaquina, Florianópolis, Santa Catarina.

\begin{tabular}{|c|c|c|c|c|c|c|c|c|}
\hline \multirow{2}{*}{ Estações } & \multicolumn{4}{|c|}{1997} & \multicolumn{4}{|c|}{1998} \\
\hline & Verão & Outono & Inverno & Primavera & Verão & Outono & Inverno & Primavera \\
\hline Verão & - & 1,01 & 0,97 & 1,01 & - & 1,01 & 0,99 & 1,01 \\
\hline Outono & - & - & 0,98 & 1,01 & - & - & 0,99 & 0,96 \\
\hline Inverno & - & - & - & 1,01 & - & - & - & 0,96 \\
\hline
\end{tabular}

De modo geral, algumas espécies usadas por C. morschi também o são por operárias de Acromyrmex striatus na mesma localidade: além de Stylosanthes viscosa, têm-se D odonaea viscosa (L.) Jacq. (Sapindaceae), Noticastrum malmei Zardini (Compositae), Paepalanthus polyanthus (Bong.) Kunth (Eriocaulaceae), Panicum sp. e Paspalum sp. (Gramineae), Polygala cyparissias St.-Hill. \& Moquin (Polygalaceae) e Stylosanthes viscosa (Leguminosae) (Lopes 2005).

Diferente das variações sazonais de uso de substratos verificados por Leal \& Oliveira (2000) para diversas espécies de Attini em cerrado, C. morschi não exibiu mudanças sazonais de itens forrageados, com exceção da comparação entre outono e inverno de 1998 (Tab. II).

O uso de material exaurido do lixo de Acromyrmex e de Atta por parte de outras espécies de formigas tem sido relatado, mas merece estudos mais aprimorados. Segundo M ARTIN \& WEBER (1969), o fungo associado aos Attini utiliza cerca de 45,0\% da energia total das folhas verdes trazidas por essas cortadeiras ou, em outras palavras, ainda resta muito material energético a ser trabal hado por outras espécies de formigas. Dentre os Attini já foi verificado que Cyphomyrmex transversus Emery, 1894 usa o material do lixo de Acromyrmex lundi (Guérin, 1838) (BucheR 1974) e Mycocepurus spp. utilizam sementes de plantas de cerrado de lixos de Acromyrmex sp. (LeAL \& Oliveira 1998).

Assim, o comportamento observado em campo, de remoção de substrato em lixo de Acromyrmex striatus, uma espécie de cortadeira muito comum na área de restinga da Praia da Joaquina (LoPEs 2005), parece ser bem generalizado, tanto para Attini como para outras espécies, onívoras ou granívoras.

Um outro aspecto da ecologia de forrageio de Cyphomyrmex que merece mais estudos diz respeito às fontes de energia usadas pelas operárias. HowARD (1991) e WetTERER (1994) sugeriram que Attini "inferiores", ao contrário das cortadeiras de material vegetal fresco, devem conseguir sua energia de outras fontes que não dos detritos forrageados. MuraKamı \& HIGASHI (1997) apontaram que as operárias mais vel has de Cyphomyrmex rimosus (Spinola, 1853) que saem para forragear podem se alimentar de néctar e líquidos das plantas (fitofagia), enquanto que as operárias mais jovens e principalmente a rainha e as larvas têm no fungo simbiôntico a sua fonte de alimento (micofagia). Foi observado em campo que operárias de C. morschi podem ficar próximas às operárias de Acromyrmex striatus quando em atividade de corte e, assim, podem aproveitar o líquido liberado pelas fo-
Ihas verdes quando cortadas. Esse tipo de registro deve ser bem comum (em campo, no presente trabalho, ou em laboratório (Murakamı \& HıgASH 1997)) e merece acompanhamentos e quantificações futuras.

Do ponto de vista do papel ecológico desempenhado, os Attini “inferiores” podem ser considerados detritófagos, já que recolhem detritos vegetais ou animais, enquanto que as cortadeiras seriam consideradas consumidoras primárias, em função do efeito causado na vegetação (BuCHeR 1974, FowLER et al. 1991).

O fato de levar principalmente material de origem vegetal para o cultivo do fungo simbiôntico já pode ser considerado um fator importante para sugerir que Cyphomyrmex tenha uma posição filogenética intermediária dentro da tribo Attini. Essa posição intermediária do gênero dentro da tribo também é compartilhada do ponto de vista da morfologia das larvas (Schultz \& Meier 1995).

\section{AGRADECIMENTOS}

A Harold G. Fowler (Instituto de Biociências, UNESP, Rio Claro), pela orientação e à CAPES, pela concessão de bolsa durante o período de Doutorado.

\section{REFERÊNCIAS BIBLIOGRÁFICAS}

Bonnet, A. \& B.C. Lopes. 1993. Formigas de dunas e restingas da Praia da Joaquina, Ilha de Santa Catarina, SC (Insecta: Hymenoptera). Biotemas 6 (1): 107-114.

Brandão, C.R.F. \& A.J.M AYhé-Nunes. 2001. A new fungus-growing ant genus, Mycetagroicus gen. $n$., with the description of three new species and comments on the monophyly of the Attini (Hymenoptera: Formicidae). Sociobiology 38 (3B): 639-665.

BROWER, J.E.; J.H. ZAR \& C.N. von ENDE. 1998. Field and laboratory methods for general ecology. Boston, WCB McGraw-Hill, $4^{\text {th }}$ ed., XI+273p.

BUCHER, E.H. 1974. Observaciones ecológicas sobrelos artropodos del bosque chaqueño de Tucumán. Revista de Facultad de Ciências Exatas, Físicas y Naturales de Córdoba (Nueva Série) Biología 1: 35-122.

Castellani, T.T.; S. Vieira \& K.Z. Scherer. 1996. Contribuição ao conhecimento da distribuição espacial de Paepalanthus polyanthus (Bong.) Kunth (Eriocaulaceae) em áreas de baixada úmida de dunas. Acta Botanica Brasilica 10 (1): 25-36.

Revista Brasileira de Zoologia 24 (1): 52-56, março 2007 
Currie, C.R.; J.A. Scott; R.C. Summerbell \& D. Malloch. 1999. Fungus-growing ants use antibiotic-producing bacteria to control garden parasites. Nature 398: 701-704.

Fowler, H.G.; L.C. Forti; C.R.F. Brandão; J.H.C. Delabie \& H.L. VASCONCELOS. 1991. ECologia nutricional de formigas, p. 131223. In: A.R. PANIZZI \& J.R.P. PARRA (Eds). Ecologia nutricional de insetos e suas implicações no manejo de pragas. São Paulo, Editora Manole \& CNPq, 359p.

Fowler, H.G.; L.C. Forti; V. Pereira-da-Silva \& N.B. Saes. 1986. Economics of grass-cutting ants, p. 18-35. In: C.S. LofGREN \& R.K. VANDER MeER (Eds.). Fire ants and leaf-cutting ants biology and management. Boulder, Westview Press, 435p.

Hölldobler, B. \& E.O. Wilson. 1990. The ants. Cambridge, The Belknap Press of the Harvard University Press, 732p.

HowARD, J.J. 1991. Resource quality and cost in the foraging of leaf-cutter ants, p. 42-50. In: C.R. HuxLEY \& D.D. CutLeR (Eds.). Ant-plant interactions. Oxford, Oxford University Press, $601 p$.

KEMPF, W.W. 1964. A revision of the Neotropical fungus-growing ants of the genus Cyphomyrmex Mayr. Part I: group of strigatus Mayr (Hym., Formicidae). Studia Entomologica 7 (1-4): 1-44.

KusNeZov, N. 1963. Zoogeografia de las hormigas en Sudamérica. Acta Zoologica Lilloana 19: 25-186.

LEAL, I.R. \& P.S. OliveiRA. 1998. Interactions between fungusgrowing ants (Attini), fruits and seeds in cerrado vegetation in Southeast Brazil. Biotropica 30 (2): 170-178.

LeAL, I.R. \& P.S. Oliveira. 2000. Foraging ecology of attine ants in a Neotropical savanna: seasonal use of fungal substrate in the cerrado vegetation of Brazil. Insectes Sociaux 47 (4): 376-382.

LOPES, B.C. 2005. Recursos vegetais usados por Acromyrmex striatus (Roger) (Hymenoptera, Formicidae) em restinga da Praia da Joaquina, Florianópolis, Santa Catarina, Brasil. Revista Brasileira de Zoologia 22 (2): 372-382.

Martin, M.M. \& N.A. Weber. 1969. The cellulose-utilizing capability of the fungus cultured by the attine ant, Atta colombica tonsipes. Annals of the Entomological Society of America 62 (6): 1386-1387.

MayhÉ-Nunes, A.J \& \& K. Jaffé. 1998. On the biogeography of Attini
(Hymenoptera: Formicidae). Ecotropicos 11 (1): 45-54.

Mueller, U.G. \& W.T. Wcislo. 1998. Nesting biology of the fungus-growing ant Cyphomyrmex longiscapus Weber (Attini, Formicidae). Insectes Sociaux 45 (2): 181-189.

Murakamı, T. \& S. HIgASHı. 1997. Social organization in two primitiveattineants, Cyphomyrmex rimosus and Myrmicocrypta ednaella, with reference to their fungus substrates and food sources. Journal of Ethology 15 (1): 17-25.

RockWood, L.L. 1976. Plant selection and foraging patterns in two species of leaf-cutting ants (Atta). Ecology 57 (1): 4861.

Santos, C.R.; N.O. Horn Filho \& T.T. Castellani. 1997. Estudo geológico e ambiental da Praia da Joaquina, p. 259-270. In: R.S. AbsalÃo \& A.M. Esteves (Eds). Oecologia Brasiliensis. Ecologia de praias arenosas do litoral brasileiro. Rio de Janeiro, Editora da UFRJ, vol 3.

Schultz, T.R. \& R. MeleR. 1995. A phylogenetic analysis of the fungus-growing ants (Hymenoptera: Formicidae: Attini) based on morphological characters of the larvae. Systematic Entomology 20 (3): 337-370.

TORRES, J.A. 1989. The status of the fungus-grower ants (Hyme noptera: Formicidae) in Puerto Rico and adjacent islands. Journal of Agriculture of the University of Puerto Rico 73 (4): 401-403.

Von Ihering, H. 1894. Die Ameisen von Rio Grande do Sul. Berliner Entomologische Zeitschrift 39 (3): 321-446.

W ALLER, D.A. 1989. Foraging behavior of Trachymyrmex turrifex Wheeler (Formicidae: Attini). The Southwestern Naturalist 34 (2): 271-275.

Weber, N.A. 1972. Gardening ants, the Attines. Memoirs of the American Philosophical Society 92: 1-146.

Wetterer, J.K. 1994. Nourishment and evolution in fungusgrowing ants and their fungi, p. 309-328. In: J.H. HuNT \& C.A. Nalepa (Eds). Nourishment and evolution in insect societies. Boulder, Westview Press, 449p.

WiRTH, R.; W. Beyschlag; R.J. Ryel \& B. Hölldobler. 1997. Annual foraging of the leaf-cutting ant Atta colombica in a semideciduous rain forest in Panama. Journal of Tropical Ecology 13: 741-757.

Recebido em 21.III.2006; aceito em 23.II.2007. 